\title{
Isolation, identification and immunomodulatory effect of a peptide from Pseudostellaria heterophylla protein hydrolysate
}

Qian Yang a, Xixi Cai a , Muchen Huang a, Xuan Chen a, Yongqi Tian a, Guiqing Chen

b, Mingfu Wang c, Shaoyun Wang a,*, Jianbo Xiao ${ }^{\text {d,* }}$

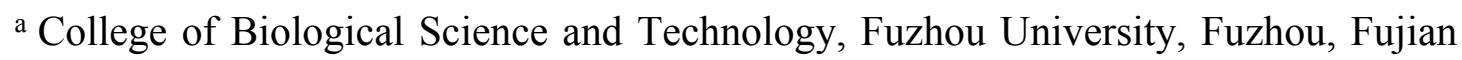
355300, China

${ }^{\mathrm{b}}$ Zhongshi Beishan (Fujian)Wine Co., LTD, Zherong, Fujian 350108, China

c School of Biological Sciences, The University of Hong Kong, Pokfulam Road, Hong Kong

${ }^{\mathrm{d}}$ Institute of Food Safety and Nutrition, Jinan University, Guangzhou 510632, China

170884247@qq.com

caixx_0123@163.com

913122340@qq.com

603256409@qq.com

601197022@qq.com

shywang@fzu.edu.cn

mfwang@hku.hk

* Corresponding author.

E-mail: shywang@fzu.edu.cn (S. Y.Wang); jianboxiao@yahoo.com (J. B. Xiao) 
Table S1 RT-PCR primer sequences used in our study.

\begin{tabular}{lll}
\hline & Forward primer & Reverse primer \\
\hline TLR2 & CCTGTTGATCTTGCTCGTAGGTGCC & TTCATCCAAGGGCCACTCCAGGTAG \\
TLR4 & TTCTTCTCCTGCCTGACACC & ATCCAGCCACTGAAGTTCTG \\
$\beta$-actin & GAGACCTTCAACCCAGCC & AATGTCACGCACGATTTCCC \\
\hline
\end{tabular}


Table S2 Peptide sequences of F7, F14, F18 and F19.

\begin{tabular}{|c|c|c|c|}
\hline Fraction & Sequences & Mass & Length \\
\hline \multirow[t]{26}{*}{7} & EGDEDGNGDDDDDDGDDDDDDDDDE & 2702.7251 & 25 \\
\hline & YGPSSYYGYG & 949.3817 & 9 \\
\hline & CPGCGSD & 637.1836 & 7 \\
\hline & PPAAHCC & 697.2676 & 7 \\
\hline & PVPSKK & 654.4064 & 6 \\
\hline & PPCPP & 509.2308 & 5 \\
\hline & GHMAGCMS & 792.2717 & 8 \\
\hline & FDFSSNSF & 949.3817 & 8 \\
\hline & DPPGY & 547.2278 & 5 \\
\hline & FYGNSF & 733.3071 & 6 \\
\hline & $\mathrm{HCSDH}$ & 597.1965 & 5 \\
\hline & EAPAESPAGPAAAP & 1234.5829 & 14 \\
\hline & HCCTS & 549.1676 & 5 \\
\hline & FDVINAF & 824.4068 & 7 \\
\hline & HCSHD & 597.1965 & 5 \\
\hline & PELIMFR & 904.484 & 7 \\
\hline & GQILCCE & 764.3197 & 7 \\
\hline & CPPSP & 499.2101 & 5 \\
\hline & CHGGY & 535.1849 & 5 \\
\hline & AAGGY & 437.191 & 5 \\
\hline & EAPAGSSD & 732.2926 & 8 \\
\hline & LLLLALFF & 948.6048 & 8 \\
\hline & ACAAGSS & 565.2166 & 7 \\
\hline & RIGAW & 601.3336 & 5 \\
\hline & RLGAW & 601.3336 & 5 \\
\hline & SSCAM & 497.1614 & 5 \\
\hline \multirow[t]{10}{*}{14} & PAAKLVLHL & 960.612 & 9 \\
\hline & HSESVHLC & 910.3967 & 8 \\
\hline & SMAFSFDAF & 1021.4215 & 9 \\
\hline & FDHELSEDA & 1061.4302 & 9 \\
\hline & FDHEISEDA & 1061.4302 & 9 \\
\hline & HSEDFSAF & 938.377 & 8 \\
\hline & QEPGSSHECL & 1085.4447 & 10 \\
\hline & DLSYNF & 757.3282 & 6 \\
\hline & YFGYT & 649.2748 & 5 \\
\hline & STSSSHVSE & 919.3883 & 9 \\
\hline \multirow[t]{5}{*}{18} & YAHVDFFMG & 1085.464 & 9 \\
\hline & SEQGKFFLT & 1055.5287 & 9. \\
\hline & FCFCCK & 749.2699 & 6 \\
\hline & FDSVFDVHS & 1051.4611 & 9 \\
\hline & FIGSSF & 656.317 & 6 \\
\hline
\end{tabular}




\begin{tabular}{|c|c|}
\hline NSPAATTM & 791.3484 \\
\hline TVVDSIGN & 803.4025 \\
\hline GHAAAETA & 726.3297 \\
\hline DAFSFFGE & 918.3759 \\
\hline DIYGLISS & 866.4385 \\
\hline YVSHE & 633.2758 \\
\hline CHPSFIGY & 922.4007 \\
\hline KSSTT & 522.265 \\
\hline VDVWWGLVEGK & 1286.6659 \\
\hline DLYGLSLSG & 923.46 \\
\hline DIACGPSH & 798.333 \\
\hline QYKTTG & 696.3442 \\
\hline VSMGGNA & 634.2745 \\
\hline PNGSCC & 579.1781 \\
\hline ELGGTF & 622.2962 \\
\hline EIGGTF & 622.2962 \\
\hline FESPETHG & 902.377 \\
\hline PAPAPGK & 636.3595 \\
\hline PCGAAFLAETD & 1093.475 \\
\hline DVWWGLVEGK & 1187.5975 \\
\hline YVYSV & 629.306 \\
\hline PPGPGP & 520.2645 \\
\hline CGNNGHN & 714.2504 \\
\hline PCNHSFR & 859.3759 \\
\hline LFCTS & 569.252 \\
\hline PAADYG & 592.2493 \\
\hline GWGGAGGAGG & 745.3143 \\
\hline LGACL & 475.2465 \\
\hline QRFRALASAR & 1174.6682 \\
\hline WGMKN & 634.2897 \\
\hline VACQCEF & 798.304 \\
\hline ENGCMN & 666.2101 \\
\hline APACC & 463.1559 \\
\hline VVKKA & 543.3744 \\
\hline RGPPP & 522.2914 \\
\hline KSTKK & 590.3751 \\
\hline DLYGLTGVS & 923.46 \\
\hline DANDANM & 749.265 \\
\hline VNICALI & 744.4204 \\
\hline $\mathrm{NNDCH}$ & 601.1915 \\
\hline HCKLV & 598.3261 \\
\hline DLGIM & 547.2676 \\
\hline SLNTT & 534.265 \\
\hline VSSGAA & 490.2387 \\
\hline
\end{tabular}




\begin{tabular}{|c|c|c|c|}
\hline & AAASP & 415.2067 & 5 \\
\hline & MSLTDP & 662.2946 & 6 \\
\hline & GSLDS & 477.2071 & 5 \\
\hline & APTAPP & 552.2907 & 6 \\
\hline & MPVHS & 569.2632 & 5 \\
\hline & DAVLM & 547.2676 & 5 \\
\hline & WLFAD & 650.3064 & 5 \\
\hline & CPGCSS & 552.1672 & 6 \\
\hline & FFLKNTPESG & 1138.5658 & 10 \\
\hline & VAFEQCC & 798.304 & 7 \\
\hline & DPSGNDENA & 917.3362 & 9 \\
\hline & ATCNGGPAE & 818.3229 & 9 \\
\hline & RGVGV & 486.2914 & 5 \\
\hline & HHPNS & 590.2561 & 5 \\
\hline & TTEGM & 537.2104 & 5 \\
\hline & FAAMN & 552.2366 & 5 \\
\hline & LTWCS & 608.2628 & 5 \\
\hline & TMFTA & 569.252 & 5 \\
\hline & AVSGGGGS & 590.266 & 8 \\
\hline & STYTA & 541.2384 & 5 \\
\hline & VRPNALTY & 932.5079 & 8 \\
\hline & FDASFLGY & 918.4123 & 8 \\
\hline \multirow[t]{22}{*}{19} & VDVWWGLVEGK & 1286.6659 & 11 \\
\hline & FDHVSLKES & 1060.5189 & 9 \\
\hline & SVFDTGFFGE & 1104.4763 & 10 \\
\hline & FDVCFSAF & 934.3895 & 8 \\
\hline & AAGPPP & 508.2645 & 6 \\
\hline & QSPVVVVAFDATW & 1417.7241 & 13 \\
\hline & VDVWFKNVER & 1290.672 & 10 \\
\hline & DAFSFFGE & 918.3759 & 8 \\
\hline & GGAGGAPF & 632.2918 & 8 \\
\hline & YEDDGAGAG & 853.309 & 9 \\
\hline & MLASVVDKPSQML & 1417.731 & 13 \\
\hline & VVKAK & 543.3744 & 5 \\
\hline & GYICMTFALC & 1120.4756 & 10 \\
\hline & CPAAK & 488.2417 & 5 \\
\hline & HGPPGT & 564.2656 & 6 \\
\hline & HSDTT & 559.2238 & 5 \\
\hline & TTAAVDS & 663.3075 & 7 \\
\hline & APKGY & 534.2802 & 5 \\
\hline & MPGGTP & 558.2472 & 6 \\
\hline & YISYL & 657.3373 & 5 \\
\hline & SQFGEIT & 780.3654 & 7 \\
\hline & FDAFSFVS & 918.4123 & 8 \\
\hline
\end{tabular}




\begin{tabular}{lcc} 
FGEHPGEF & 918.3871 & 8 \\
THKGF & 588.302 & 5 \\
GYGLL & 521.2849 & 5 \\
LPIPQIVAKEG & 1163.6914 & 11 \\
NCSNAASSS & 839.3079 & 9 \\
DVVSPFE & 791.3701 & 7 \\
ACPGCSC & 639.1815 & 7 \\
KGSGH & 484.2394 & 5 \\
LYESAYW & 930.4123 & 7 \\
PCGACM & 580.1808 & 6 \\
FGFESDGLAG & 998.4344 & 10 \\
CAATTT & 566.237 & 6 \\
DSTPSSSSSPSD & 1152.4419 & 12 \\
YVSDF & 629.2697 & 5 \\
SDFTTSSFPD & 1102.4454 & 10 \\
DSDLEPIMSD & 1120.4594 & 10 \\
GAQCCSD & 682.2051 & 7 \\
CHPSFIGY & 922.4007 & 8 \\
DNPCACGS & 765.2422 & 8 \\
INGADSS & 662.2871 & 7 \\
KVVHA & 552.3384 & 5 \\
GVKFA & 520.3009 & 5 \\
VVAKK & 543.3744 & 5 \\
CPGSS & 449.158 & 5 \\
KIGYG & 536.2958 & 5 \\
LLVGV & 499.337 & 5 \\
DAFDFSAF & 918.3759 & 8 \\
WPSPF & 632.2958 & 5 \\
SSAEA & 463.1914 & 5 \\
EAVNDS & 633.2606 & 6 \\
DLGMI & 547.2676 & 5 \\
AKPSS & 488.2594 & 5 \\
VCFSN & 568.2315 & 5 \\
\hline
\end{tabular}



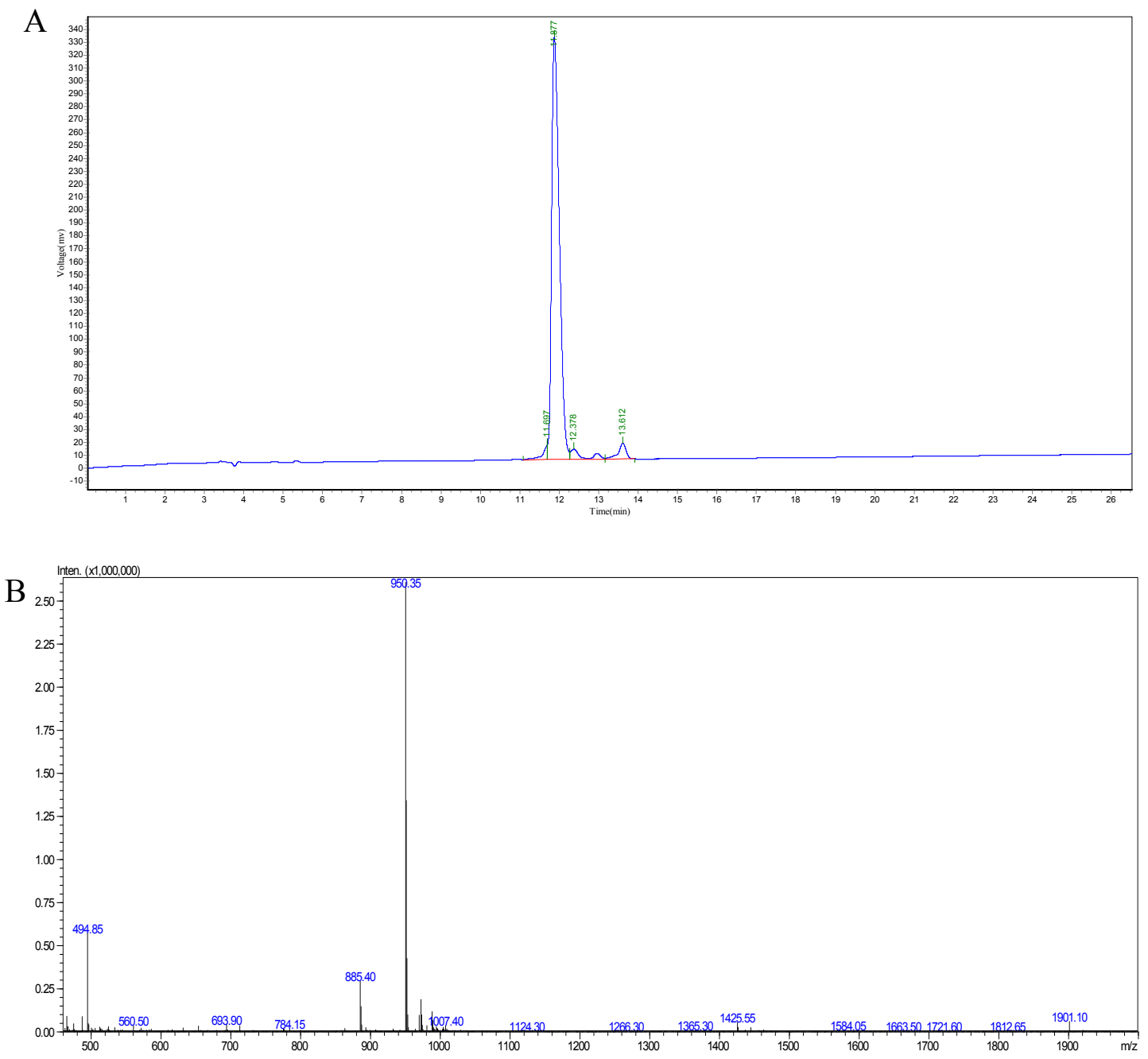

$\mathrm{C}^{\text {Intensity (\%) } \mathbf{Y} / \mathbf{G} \sqrt{\mathbf{P}}\rfloor \mathbf{S} \sqrt{\mathbf{S}} \sqrt{\mathbf{Y}} \sqrt{\mathbf{G}} \sqrt{\mathbf{Y}}\rfloor \mathbf{G}}$

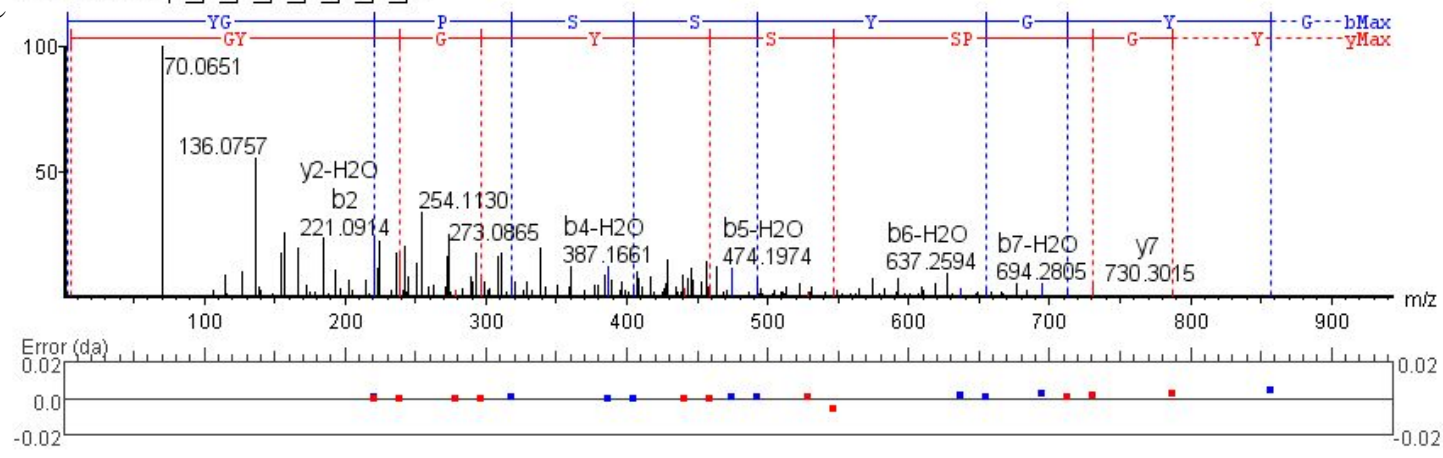

Fig. S1 HPLC (A), MS (B) and MS/MS analysis (C) of synthetic YG-9. 